Check for updates

Cite this: RSC Adv., 2019, 9, 36570

\title{
Polymer network-derived nitrogen/sulphur co- doped three-dimensionally interconnected hierarchically porous carbon for oxygen reduction, lithium-ion battery, and supercapacitor
}

\begin{abstract}
Zili Xu, ${ }^{a}$ Fangfang Zhang, ${ }^{\text {b }}$ Weiran Lin*c and Haining Zhang (D) *a
Rational design and simple synthesis of carbon-based materials with high electrocatalytic activity are essential for their practical applications in electrochemical energy conversion and storage devices. Herein, we report the synthesis of nitrogen, sulfur co-doped three-dimensional interconnected hierarchically porous carbon (NSHPC) by zinc acetate assisted pyrolysis of polymer networks. The thussynthesized NSHPC has a specific surface area of $1057 \mathrm{~cm}^{2} \mathrm{~g}^{-1}$ with the coexistence of micro- and meso-pores. As metal-free electrocatalyst, the NSHPC exhibits a promising activity towards oxygen reduction reactions as evidenced by the slightly negative shift of half-wave potential compared with commercial Pt/C catalyst. The assembled lithium ion battery using NSHPC as anode delivers the reversible capacity of 740 and $470 \mathrm{~mA} \mathrm{~h} \mathrm{~g}^{-1}$ at current densities of 2 and $5 \mathrm{~A} \mathrm{~g}^{-1}$ without performance decay after 1000 charge-discharge cycles. Moreover, the assembled supercapacitor using NSHPC as electrode has the capacitance of $203 \mathrm{~F} \mathrm{~g}^{-1}$ at $1 \mathrm{~A} \mathrm{~g}^{-1}$.
\end{abstract}

Received 20th September 2019 Accepted 25th October 2019

DOI: 10.1039/c9ra07619k

rsc.li/rsc-advances distribution, and electron transfer behaviour, accordingly leading to tuneable electrocatalytic activity. ${ }^{\mathbf{1 0 - 1 4}}$

It is generally believed that the heteroatom-doped porous carbon applied as electrode materials should have interconnected channels for fast supply and transportation of reactants and products, high void volume for storage of reactants and products, enough active sites for fast reaction kinetics, and excellent electronic conductivity for rapid electron transport. ${ }^{15}$ Heteroatoms-doped porous carbon materials have thus been extensively synthesized by using template-assisted or templatefree techniques and the according electrochemical performance has been evaluated for oxygen-involved electrochemical reactions, lithium ion batteries, supercapacitors, and solar cells as well. ${ }^{16-18}$ For example, 3D nitrogen-doped porous graphene framework derived from inter-connected nanocages exhibited good bifunctional catalytic activity toward both oxygen reduction reaction (ORR) and oxygen evolution reaction (OER) and the thus-assembled lithium-oxygen battery delivered a long cycle stability at a large current density $\left(54\right.$ cycles at $\left.1 \mathrm{Ag}^{-1}\right) \cdot{ }^{17}$ In another example, B-doped graphene sheets as anode materials displayed super-high rate and large capacity for LIBs with a capacity of $235 \mathrm{~mA} \mathrm{~h} \mathrm{~g}^{-1}$ at $25 \mathrm{~A} \mathrm{~g}^{-1} .^{18}$

Among the developed heteroatoms-doped porous carbon materials, nitrogen/sulphur-co-doped porous carbon has attracted special attention due to the existed synergetic effect between nitrogen and sulphur atoms on their electrocatalytic activity. ${ }^{19}$ Moreover, the relatively low energy required for nitrogen/sulphur co-doping compared to that for separating
${ }^{a}$ State Key Laboratory of Advanced Technology for Materials Synthesis and Processing, Wuhan University of Technology, Nr. 122 Luoshi Rd., Wuhan 430070, China. E-mail: haining.zhang@whut.edu.cn

${ }^{b}$ School of Nuclear Technology and Chemistry \& Biology, Hubei University of Science and Technology, Nr. 88 Xianning Avenue, Xianning 437100, China

'The Fundamental Industry Training Center, Tsinghua University, Beijing 100084, China.E-mail: linwr@tsinghua.edu.cn 
heteroatoms from porous carbon matrix as evidenced by theoretical calculation makes the fabrication process quite straightforward..$^{20-22}$ Nitrogen/sulphur co-doped porous carbon materials are often synthesized by template-assisted pyrolysis of organic or polymeric precursors, followed by removal of templates (typically silica). ${ }^{23}$ Despite of the straightforward synthetic approach, the yield and surface area of the final products is still limited. Thus, rational design and synthesis of nitrogen/sulphur co-doped porous carbon materials with tuneable porous structure and composition are of great importance for their practical applications as well as fundamental understanding of their electrochemical properties.

Inspired by the advantages of hierarchical materials, we report the synthesis of nitrogen/sulphur co-doped three dimensionally interconnected hierarchically porous carbon (NSHPC) by pyrolysis of pre-synthesized polymer networks with the well-distributed silica and zinc acetate templates. It is expected that the application of polymer networks as precursor can result in high yield of final carbon-based materials and can lead to the well-distribution of templates. As templates, silica nanoparticles provide mesopores in the final products which facilitate mass transport during electrochemical process whereas zinc acetate molecules can be reduced and gasified under pyrolysis to generate micropores which are beneficial for the increase in surface area and ion transport. ${ }^{24,25}$ Electrochemical performance of the thus-synthesized NSHPC materials as electrode with respect to oxygen reduction reaction (ORR), lithium ion battery, and supercapacitor was further evaluated and the results demonstrate that synthesized NSHPC materials are multifunctional and exhibit relatively wide applicability in electrochemical energy conversion and storage devices.

\section{Experimental section}

\section{Synthesis of NSHPC}

To a solution containing $3 \mathrm{~mL}$ of deionized water, $10 \mathrm{~mL}$ of 1vinylimidazole (1-VIm), $255.3 \mathrm{mg}$ of $N, N^{\prime}$-methylenebisacrylamide (MBA), and $1.266 \mathrm{~g}$ of zinc acetate $\left(\mathrm{Zn}(\mathrm{Ac})_{2}\right)$, $1.25 \mathrm{~g}$ of silica template (30 $\mathrm{nm}$ and surface area of $220 \mathrm{~m}^{2} \mathrm{~g}^{-1}$ ) was added, followed by $10 \mathrm{~min}$ ultrasonic treatment to form a stable dispersion. $5 \mathrm{~g}$ of an aqueous solution of ammonium persulfate (APS $7.5 \mathrm{wt} \%$ ) was then added and reacted at $85{ }^{\circ} \mathrm{C}$ for $1 \mathrm{~h}$ under magnetic stirring. The formed polymer network was transferred to an oven and was dried at $90{ }^{\circ} \mathrm{C}$ for $12 \mathrm{~h}$. The resulting polymer network precursor were then carbonized in a tube furnace under argon flow with a ramp rate of $5{ }^{\circ} \mathrm{C} \mathrm{min}^{-1}$ and held at $1000{ }^{\circ} \mathrm{C}$ for $1 \mathrm{~h}$. The carbonized material was dispersed in $20 \mathrm{~mL}$ of $40 \% \mathrm{HF}$ aqueous solution to remove the silica templates and residual zinc. After extensively washing with deionized water until a neutral $\mathrm{pH}$ reached, the final product was dried under vacuum overnight at $90{ }^{\circ} \mathrm{C}$ in a vacuum oven. The controlling samples with $0 \mathrm{~g}, 1 \mathrm{~g}$ and $1.5 \mathrm{~g}$ of silica templates (denoted as NSHPC-0Silica, NSHPC-1Silica and NSHPC-1.5Silica, respectively), and the controlling sample without crosslinking agents (denoted as NSHPC-0CA), and the controlling sample with $1 \mathrm{~g}$ and $1.5 \mathrm{~g}$ of zinc acetate (denoted as
NSHPC-1Zn and NSHPC-1.5Zn, respectively) in the initial materials were prepared by the same procedure except the different chemical gradient. Moreover, the NSHPC-900 refers to the sample carbonized at $900{ }^{\circ} \mathrm{C}$ under the same condition.

\section{Characterization}

Morphology and microstructure of the synthesized samples were characterized with scanning electron microscope (SEM JEM-7500F) and high-resolution transmission electron microscopy (HRTEM JEM-2100F). The XRD measurements were taken on D/MAX-RB RU-200B with $\mathrm{Cu} \mathrm{K} \alpha$ radiation $(\lambda=1.5406 \AA)$. Raman spectra were conducted on LabRamHR800 (resolution of $4 \mathrm{~cm}^{-1}$ ) to investigate the crystallographic structure and degree of graphitization respectively. The XPS (VG Multilab2000X) measurement was performed to analyze the chemical state of atoms near the surface quantitatively. $\mathrm{N}_{2}$ adsorption/ desorption isotherms were measured with a Micromeritics 3Flex 3500. Prior to measurement, samples were degassed under vacuum overnight to remove physically adsorbed gases. Surface area and pore size distribution were calculated from the adsorption-desorption isotherm of nitrogen. To determine the Lewis basic sites of the synthesized materials, $\mathrm{CO}_{2}$ adsorption was performed on a temperature-programmed desorption (TPD) apparatus TP-5080 (TianJin XianQuan, China). The detailed process for $\mathrm{CO}_{2}$ adsorption measurements was described elsewhere.

\section{Electrochemical measurements}

All electrochemical measurements of oxygen reduction reaction (ORR) including cyclic voltammetry (CV) and linear sweep voltammetry (LSV) were carried out on a CHI 660E electrochemical workstation using rotating disk electrode at room temperature. A conventional three-electrode system was applied with platinum black electrode and SCE as the counter and reference electrodes respectively. The working electrodes were prepared by casting $20 \mu \mathrm{L}$ of the catalyst ink $\left(5 \mathrm{mg} \mathrm{mL} \mathrm{m}^{-1}, 780 \mu \mathrm{L}\right.$ of isopropanol $+200 \mu \mathrm{L}$ of water $+20 \mu \mathrm{L}$ of Nafion) onto the glassy carbon disk electrodes $(5.0 \mathrm{~mm}$ in diameter). The tests were carried out in $\mathrm{O}_{2} / \mathrm{N}_{2}$-saturated $0.1 \mathrm{~mol} \mathrm{~L}^{-1} \mathrm{KOH}$ solution. $\mathrm{CV}$ measurements were conducted with a scan rate of $20 \mathrm{mV} \mathrm{s}^{-1}$. LSV measurements were conducted with a scan rate of $10 \mathrm{mV}$ $\mathrm{s}^{-1}$ at different rotation rates. All potentials referring to reversible hydrogen electrodes (RHE) were converted from the test potentials versus SCE according to Nernst equation and the electron transfer number $(n)$ was evaluated according to Koutecky-Levich (K-L) equation.

To evaluate the electrochemical performance of (LIBs), Li storage tests were carried out in 2016 coin-type cells assembled inside an argon-filled glove box with both moisture and oxygen contents below $0.1 \mathrm{ppm}$. The electrolyte was $1 \mathrm{~mol} \mathrm{~L}^{-1} \mathrm{LiPF}_{6}$ dissolved in a $1: 1(\mathrm{w} / \mathrm{w})$ mixture of ethylene carbonate and dimethyl carbonate. The separator is polypropylene (PP). The working electrodes were prepared by casting the slurry onto a copper foil current collector and the slurry was a mixture of active material, acetylene black, poly(vinyl difluoride) (PVDF) binder with a mass ratio of $65: 20: 15$ and $800 \mu \mathrm{L}$ of $N$-methyl 
pyrrolidone (NMP). Then they were dried in a vacuum drying oven at $80{ }^{\circ} \mathrm{C}$ for $12 \mathrm{~h}$ to remove the solvent. The electrochemical performance was tested at various rates in the voltage range of $0.01-3.00 \mathrm{~V}\left(v s\right.$. $\left.\mathrm{Li} / \mathrm{Li}^{+}\right)$. The galvanostatic chargedischarge tests were performed using a LAND-BT testing system. Cyclic voltammetry (CV) and electrochemical impedance spectral (EIS) measurements was carried out on a CHI $660 \mathrm{E}$ electrochemical workstation.

All electrochemical measurements of supercapacitor were carried out by a CHI 660E electrochemical workstation at room temperature. A conventional three-electrode system was applied with platinum black electrode and $\mathrm{Ag} / \mathrm{AgCl}$ as the counter and reference electrodes, respectively. To prepare the testing electrode, the mixture of active material, acetylene black and polytetrafluoroethylene (PTFE) binder with a mass ratio of $8: 1: 1$ was coated onto the nickel foam current collector (with a size of around $1 \mathrm{~cm} \times 1 \mathrm{~cm}$ ), and dried at $100{ }^{\circ} \mathrm{C}$ for $12 \mathrm{~h}$ in a vacuum drying oven. The loading mass of the active material was about $1.8 \mathrm{mg} \mathrm{cm}^{-2}$ and the $6 \mathrm{~mol} \mathrm{~L}^{-1} \mathrm{KOH}$ aqueous solution served as electrolyte. Cyclic voltammetry (CV) was performed at various scan rates and galvanostatic charge/discharge curves (CP) were obtained at various current densities. The gravimetric specific capacitances $\left(C_{\mathrm{g}}\right)$ can be calculated according to the galvanostatic charge/discharge curves (CP) at different current densities.

\section{Results and discussion}

The designed nitrogen/sulphur co-doped three dimensionally interconnected hierarchically porous carbon (NSHPC) materials were synthesized through pyrolysis of pre-formed poly(1vinylimidazole) networks under existence of dual-templates of silica nanoparticles (about $30 \mathrm{~nm}$ ) and zinc acetate, followed by removal of silica templates, as schematically shown in Fig. 1. The expected advantages using polymer network as precursor include high yield of final product, well-distribution of templates, and easy formation of interconnected threedimensional carbon frameworks. During pyrolysis process, zinc acetate molecules can be first reduced to zinc and further gasification of zinc leads to the formation of micropores in the final NSHPC products. ${ }^{26}$ After removal of silica templates (about $30 \mathrm{~nm}$ ), meso-pores can be thus generated in the final products.

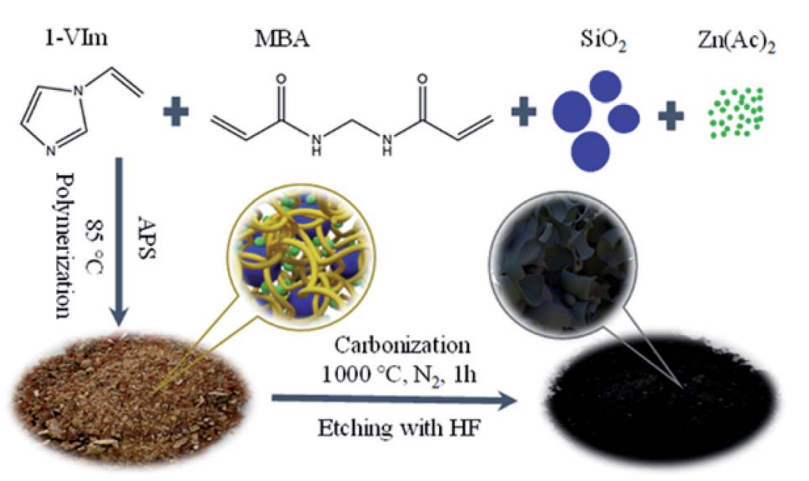

Fig. 1 Schematic illustration of the synthesis of NSHPC.
In addition, both the applied monomer 1-vinylimidazole (1VIm) and crosslinking agent $N, N^{\prime}$-methylenebisacrylamide (MBA) are nitrogen and carbon sources whereas the initiator molecules of ammonium persulfate (APS) provide sulphur atoms in the final NSHPC products.

The morphology and microstructure of the formed NSHPC were first examined by scanning electron microscopy (SEM) and transmission electron microscopy (TEM). The hollow, spherical shape porous with a diameter of about $25 \mathrm{~nm}$ (mesopores) generated by removal of silica templates are uniformly distributed inside the material, as shown in SEM image (Fig. 2a) and TEM image (Fig. 2b). The lattice fringes in high-resolution TEM image (Fig. 2c) suggest the existence of graphitic carbons layers in the synthesized NSHPC samples, which is beneficial to the conductivity of the material. For comparison, TEM image of the sample without addition of zinc acetate for carbonization process (denoted as NS-PC) was shown in Fig. 2d. It can be seen that NSHPC possesses more defects and holes in the carbon layer compared to the sample of NS-PC. Energy dispersive spectroscopy (EDS) in Fig. 2e and f reveals the presence of $\mathrm{C}, \mathrm{N}$ and $\mathrm{S}$, indicating the successful incorporation of $\mathrm{N}, \mathrm{S}$ atoms into the carbon framework and the homogeneous distribution of $\mathrm{N}$, $\mathrm{S}$ atoms on the surface of the NSHPC.

Fig. 3a shows the X-ray diffraction (XRD) pattern of NSHPC and NS-PC. The observed diffraction peaks at $2 \theta$ of $24.48^{\circ}$ and $44.28^{\circ}$ are the characteristic (002) and (101) diffraction peaks of graphitic carbon, indicating that the formed carbon layers are graphitic carbons even after N/S-co-doping. ${ }^{27}$ Moreover, the diffraction peaks of NSHPC is wider than those of NS-PC, indicating that the formed graphitic carbons of NSHPC are disorderly oriented with large interlayer spacing and defectrich. Fig. $3 \mathrm{~b}$ displays the nitrogen adsorption-desorption isotherms of both NSHPC and NS-PC samples. Type-IV isotherms with a distinctive hysteresis loop were observed in the high relative pressure region for both samples of NSHPC and NS-PC, indicating the existence of mesopores. Moreover, NSHPC exhibits a significant tail in the low relative pressure region of the nitrogen adsorption-desorption isotherm compared to NS-PC, indicating that NSHPC possesses a large number of micropores. ${ }^{28}$ From the pore-size distributions of NSHPC and NS-PC in Fig. 3c, it is evident that both NSHPC and

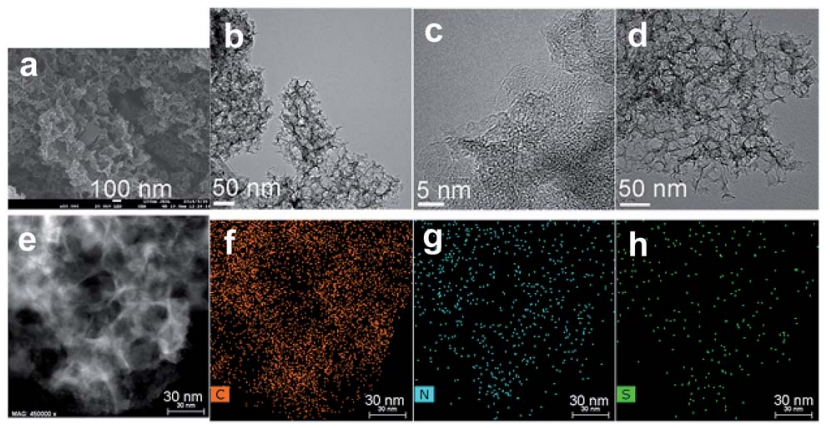

Fig. 2 SEM image (a), TEM image (b), and HRTEM (c) of NSHPC. (d) TEM image of NS-PC. (e) Typical TEM image of NSHPC, and the corresponding elemental mapping images of $\mathrm{C}(\mathrm{f}), \mathrm{N}(\mathrm{g})$, and $\mathrm{S}(\mathrm{h})$ atoms. 

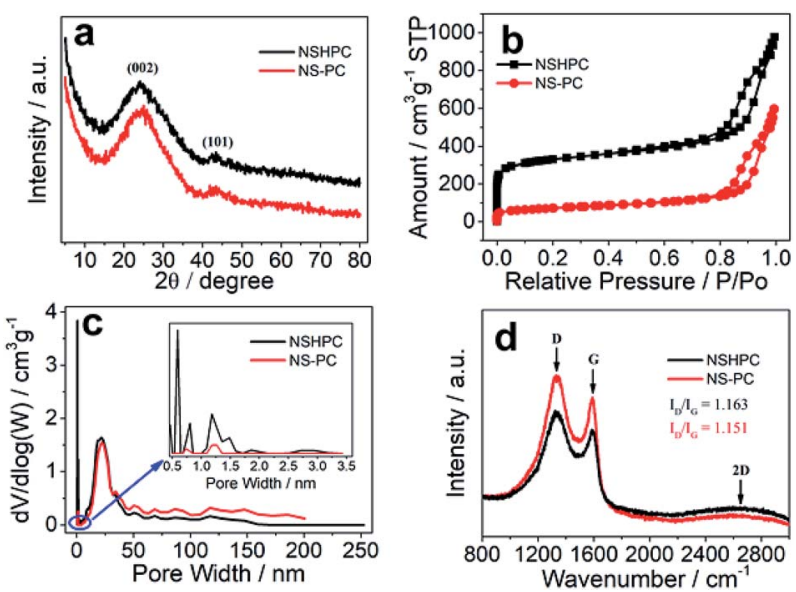

Fig. 3 X-ray diffraction patterns (a), nitrogen adsorption-desorption isotherms (b), pore size distribution (c), and Raman spectra (d) of NSHPC and NS-PC.

NS-PC have very similar mesoporous distributions centered on $25 \mathrm{~nm}$. However, the NSHPC sample possesses a distinct distribution in the microporous region $(<2 \mathrm{~nm})$ whereas no significant micropore distribution was observed for the sample of NS-PC (inset of Fig. 3c), which is consistent with the HRTEM observation. The Brunauer-Emmett-Teller (BET) specific surface area of NSHPC and NS-PC were calculated to be about $1057 \mathrm{~cm}^{2} \mathrm{~g}^{-1}$ and $244 \mathrm{~cm}^{2} \mathrm{~g}^{-1}$ respectively. It has been realized that hierarchical pore structures and high specific surface area can provide a large number of active sites and can ensure efficient mass transport, which in turn facilitates rapid ion diffusion, ion buffering, and ion storage. ${ }^{29}$ Fig. $3 \mathrm{~d}$ shows Raman spectra of NSHPC and NS-PC, which show characteristic absorption bands of D-band and G-band at about $1598 \mathrm{~cm}^{-1}$ and $1338 \mathrm{~cm}^{-1}$, respectively. Generally, the intensity ratio of the D-band to G-band $\left(I_{\mathrm{D}} / I_{\mathrm{G}}\right)$ is applied to evaluate the degree of disorder and defects in carbon materials, and a higher ratio indicates an increased amount of disorder and defects. ${ }^{30}$ The $I_{\mathrm{D}} /$ $I_{\mathrm{G}}$ ratio of NS-PC and NSHPC are about 1.151 and 1.163 respectively, indicating NSHPC has more defects than NS-PC, attributed to the existed micropores generated by zinc. ${ }^{31}$ Moreover, the relatively low and broad absorption bands of NSHPC compared to NS-PC also suggests that NSHPC possesses more disordered region and defects than NS-PC does. ${ }^{32}$ Meanwhile, there is a wide 2D peak at about $2700 \mathrm{~cm}^{-1}$ confirming its high degree of graphitization (Fig. 3d), which indicate the high electrical conductivity of NSHPC.

X-ray photoelectron spectroscopy (XPS) measurements were performed to investigate the composition and chemical status of the elements in the synthesized samples. The XPS surveys of both NSHPC and NS-PC samples (Fig. 4a) have four characteristic peaks located at $c a$. 164.02, 285.46, 399.89, and $533.09 \mathrm{eV}$, corresponding respectively to $\mathrm{S} 2 \mathrm{p}, \mathrm{C} 1 \mathrm{~s}, \mathrm{~N} 1 \mathrm{~s}$, and $\mathrm{O} 1 \mathrm{~s}$. The atomic contents of $\mathrm{N}$ and $\mathrm{S}$ are 4.68 at $\%$ and 1.58 at $\%$ for NSHPC whereas they are 4.37 at $\%$ and 1.66 at $\%$ for NS-PC, respectively. Deconvolution of high-resolution N 1s and $\mathrm{S} 2 \mathrm{p}$ spectra of NSHPC (Fig. 4b) and NS-PC (Fig. 4c) reveal the

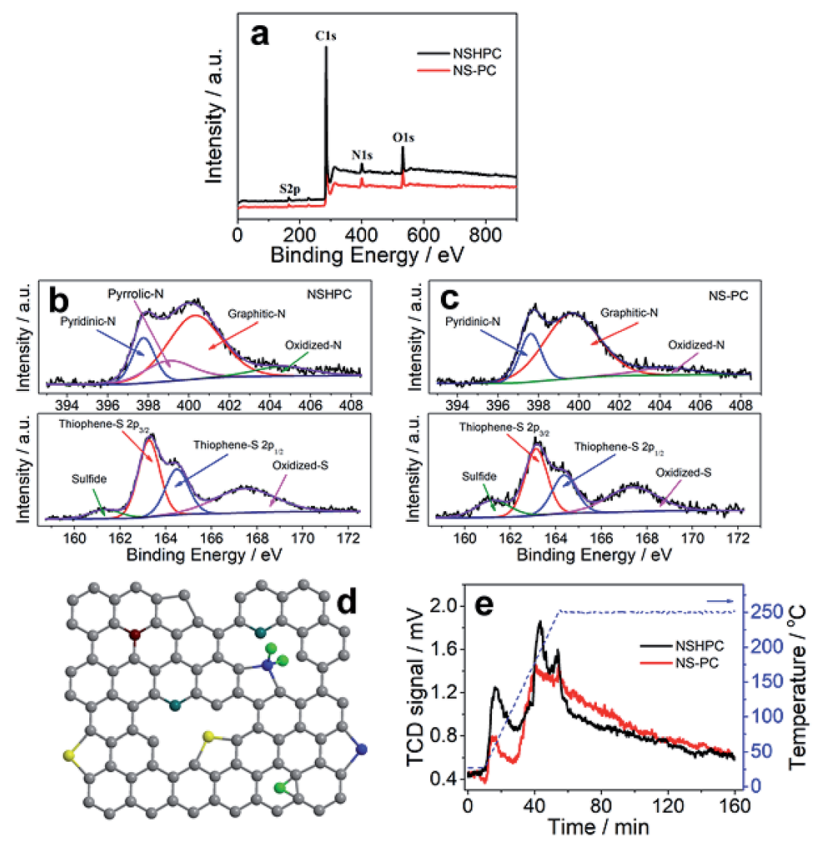

Fig. 4 (a) XPS surveys of NSHPC and NS-PC. The deconvolution of high-resolution N 1s and S 2p spectra of NSHPC (b) and NS-PC (c). (d) Schematic representation of different $\mathrm{N}$ and $\mathrm{S}$ in the framework of NSHPC. (e) Temperature-programmed $\mathrm{CO}_{2}$ desorption response of NSHPC and NS-PC. The blue dash line in (e) represents the temperature profile for $\mathrm{CO}_{2}$ desorption.

presence of pyridine-N (397.8 eV), pyrrole-N (399.1 eV), graphitic-N (400.1 eV), oxidized-N $(404.5 \mathrm{eV})$, as well as thiophenic-S (due to spin-orbit coupling) emerge for $\mathrm{S} 2 \mathrm{p}_{3 / 2}$ $(163.3 \mathrm{eV})$ and $\mathrm{S} 2 \mathrm{p}_{1 / 2}(164.5 \mathrm{eV})$, oxidized-S $(167.5 \mathrm{eV})$ and other sulphur phase for both samples. ${ }^{11,33}$ It has been reported that the doped phases have a great influence on their electrochemical performance. ${ }^{34}$ For instance, graphitic-N is regarded to improve the conductivity of materials while pyridinic-N, pyrrole-N and thiophenic-S can enhance the electrochemical activity and electrical conductivity. ${ }^{34}$ Compared to NS-PC, the enhanced proportions of pyridinic- $\mathrm{N}$ and pyrrole- $\mathrm{N}$ to the overall nitrogen atoms in NSHPC suggest the possibly more active sites for oxygen-involved electrochemical reactions. The carbon layer structural scheme of NSHPC is illustrated in Fig. $4 \mathrm{~d}$. Because of the decorated basic N/S functionalities, these doped carbon frameworks could create Lewis basic sites, which are favorable adsorbents for capturing Lewis acidic guest molecules such as oxygen and carbon dioxide. ${ }^{35-37}$ Therefore, the $\mathrm{CO}_{2}$ adsorption properties of NSHPC and NS-PC were carried out by using a temperature-programmed desorption (TPD) technique and the results were shown in Fig. 4e. Calculated from the integration of $\mathrm{CO}_{2}$ TPD responsive curves, the adsorption capacity of NSHPC and NS-PC are about 0.21 and $0.13 \mathrm{mmol} \mathrm{g}^{-1}$ respectively, demonstrating that NSHPC bears more Lewis basic sites than the NS-PC does, due to the difference in specific surface area.

The electrocatalytic activity of the prepared samples towards ORR is evaluated in $0.1 \mathrm{~mol} \mathrm{~L}^{-1} \mathrm{KOH}$ aqueous solution saturated with $\mathrm{N}_{2}$ or $\mathrm{O}_{2}$. Fig. 5a shows the CV curves of NS-PC, 

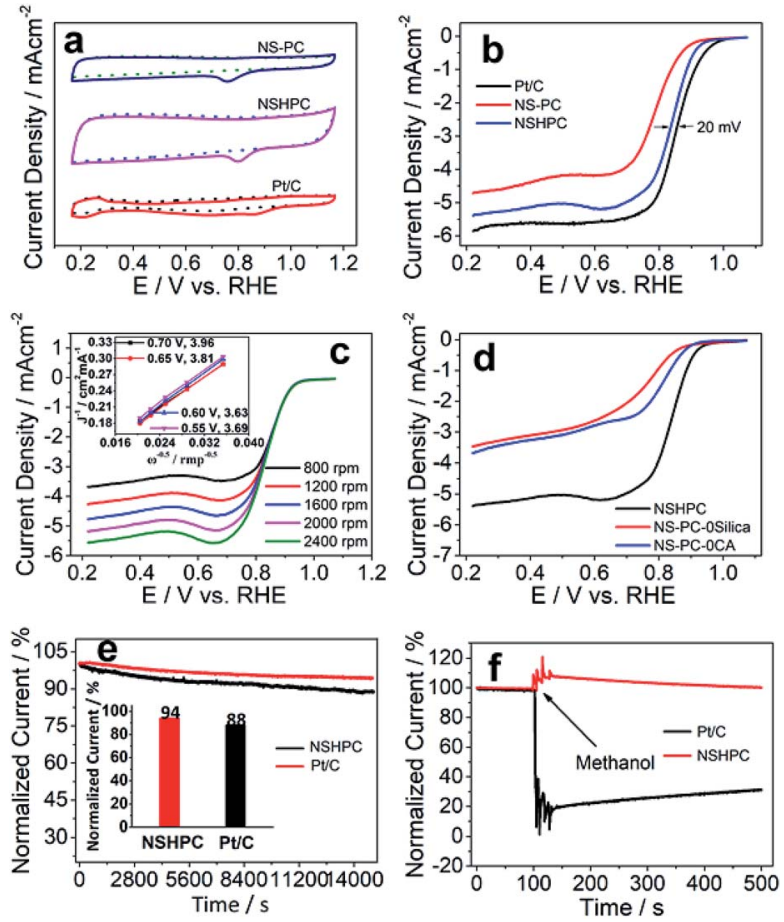

Fig. 5 CV curves (a) and LSV curves (b) of NSHPC, NS-PC, and Pt/C catalysts in $\mathrm{O}_{2}$-saturated $0.1 \mathrm{~mol} \mathrm{L^{-1 }} \mathrm{KOH}$ solution. (c) LSV curves of NSHPC at different rotation speeds and the according $K-L$ plots (inset) of NSHPC. (d) LSV curves of NSHPC, NS-PC-OSilica, and NS-PC-OCA. (e) The $i-t$ chronoamperometric response for NSHPC and Pt/C. (f) Methanol tolerance results for NSHPC and Pt/C.

NSHPC, and commercial Pt/C (20 wt $\%$ ). Clearly, the peak potential of NSHPC $\left(E_{\mathrm{p}}=0.82 \mathrm{~V}\right)$ is more positive than NS-PC $\left(E_{\mathrm{p}}=0.78 \mathrm{~V}\right)$ and is close to that of $\mathrm{Pt} / \mathrm{C}\left(E_{\mathrm{p}}=0.85 \mathrm{~V}\right)$. Fig. $5 \mathrm{~b}$ displays the LSV curves of NS-PC, NSHPC, and commercial Pt/C catalysts. It is apparent that the NSHPC exhibits higher ORR activity than the sample of NS-PC, as evidenced by the significantly positive shift of the onset potential, half-wave potential $\left(E_{1 / 2}\right)$ and the high diffusion-limited current density. In addition, the ORR activity of NSHPC is slightly worse than that of commercial Pt/C catalysts as the half-wave potential of NSHPC

Table 1 Comparison of the half-wave potentials of ORR in $0.1 \mathrm{M} \mathrm{KOH}$ aqueous solutions from literatures and this work

\begin{tabular}{lll}
\hline Materials & $E_{1 / 2} v s$. RHE & References \\
\hline GQD-MWCNT & 0.765 & 7 \\
GC-NLS & 0.73 & 10 \\
SN-CNTs & 0.81 & 11 \\
NPMC-1000 & 0.85 & 13 \\
S-graphene & 0.81 & 14 \\
SN-CF & 0.82 & 19 \\
NS-HCNS & 0.75 & 23 \\
NPC-F & 0.84 & 27 \\
ND-GLC & 0.875 & 28 \\
NSHPC & 0.833 & This work
\end{tabular}

$\left(E_{1 / 2}=0.833 \mathrm{~V}\right)$ is negatively shifted by only $20 \mathrm{mV}$ compared to commercial Pt/C $\left(E_{1 / 2}=0.853 \mathrm{~V}\right)$, but comparable or better than most of the recently reported non-metal electrocatalysts (Table 1). The results demonstrate that the three-dimensional interconnected hierarchically porous structure with large surface area are beneficial to the ORR activity due to the large number of exposed active sites. ${ }^{19}$ From the LSV curves under different rotating speed (Fig. 5c) and the according Koutechy-Levich (KL) plots (inset in Fig. 5c), the average electron transfer number in the potential range of $0.5-0.7 \mathrm{~V}$ are calculated to be above 3.6, suggesting that the dominant ORR process relies on a fourelectron transfer reaction. Furthermore, we also investigated the effect of the silica template and crosslinker agent on the ORR activity and Fig. 5d shows the comparison of LSV curves for the NSHPC, the sample without silica templates (denoted as NSPC-0Silica), and the sample without crosslinking agent (denoted as NS-PC-0CA) in precursors. It is evident that the NSHPC exhibits the best ORR activity among the tested three samples, indicating that the introduction of silica templates and the crosslinking agent in the precursors does affect the electrocatalytic properties for ORR. Apart from the catalytic activity, the stability is another important parameter for the performance of electrocatalysts. The $i-t$ chronoamperometric response curve was recorded to evaluate the stability of the synthesized catalyst, as shown in Fig. 5e. It can be seen that NSHPC has only $6 \%$ decay in relative current density after an $i-t$ test of $15000 \mathrm{~s}$, whereas Pt/C exhibits a decrease by $12 \%$, indicating that NSHPC have superior stability than Pt/C. In addition, the tolerance of catalysts against methanol was measured by recording the $i-t$ chronoamperometric response curve in the presence of methanol. As shown in Fig. 5f, the relative current density has nearly no change for NSHPC after dropping methanol for $400 \mathrm{~s}$, whereas it decreased significantly for $\mathrm{Pt} / \mathrm{C}$. The results demonstrate that the co-existence of mesopores and micropores could facilitate rapid ion diffusion, ion buffering and ion storage, and thus guaranteeing full utilization of porous networks.

To further understand how the introduced reagents for the formation of pores affect the electrocatalytic activity of the final carbon-based materials for ORR, samples with the different amount of silica template and zinc acetate were prepared and the according LSV curves recorded from in $\mathrm{O}_{2}$-saturated $0.1 \mathrm{~mol} \mathrm{~L}^{-1} \mathrm{KOH}$ aqueous solution were displayed in Fig. 6a and b. It can be seen that there exited an intermediate amount of the added amount for both silica and zinc acetate, at which the synthesized material exhibited the highest electrocatalytic activity for ORR as evidenced by the polarization curves. This could be explained that the catalytic reaction is affected by both mass transfer of reagents and the electron transfer. The increased number of pores could enhance mass transfer of electrolyte (mesopores) and oxygen (micropores) but may affect electron transfer negatively whereas the small number of pores could weaken the mass transfer of electrolytes and oxygen molecules. In addition, the effect of carbonization temperature on the electrocatalytic properties was also evaluated, as shown in Fig. 6c. It is evident that the NSHPC sample carbonized at $1000{ }^{\circ} \mathrm{C}$ exhibited the better electrocatalytic performance for 


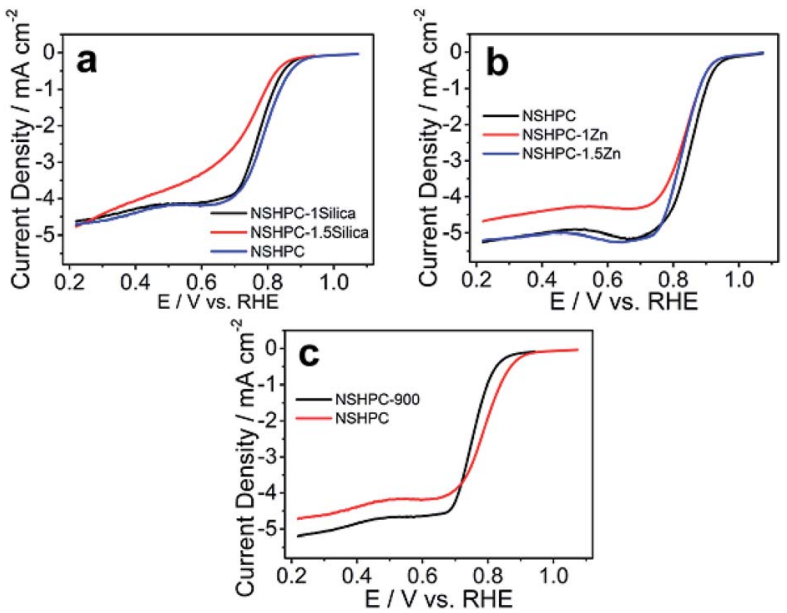

Fig. 6 LSV curves of the synthesized materials with different amounts of silica templates (a), zinc acetate (b), and different carbonization temperatures (c) as indicated in the according figure.

ORR than the sample carbonized at $900{ }^{\circ} \mathrm{C}$ (NSHPC-900) possibly due to the enhanced electric conductivity.

Lithium storage properties of NSHPC are evaluated by using standard coin-type cells. Fig. 7a shows the first three CV curves of NSHPC anode at scan rate of $0.1 \mathrm{mV} \mathrm{s}^{-1}$. Several irreversible peaks were observed during the first cathodic scan. The peak at about $0.6 \mathrm{~V}$ is assigned to the formation of a solid electrolyte interphase (SEI) layer on the surface of the electrode caused by
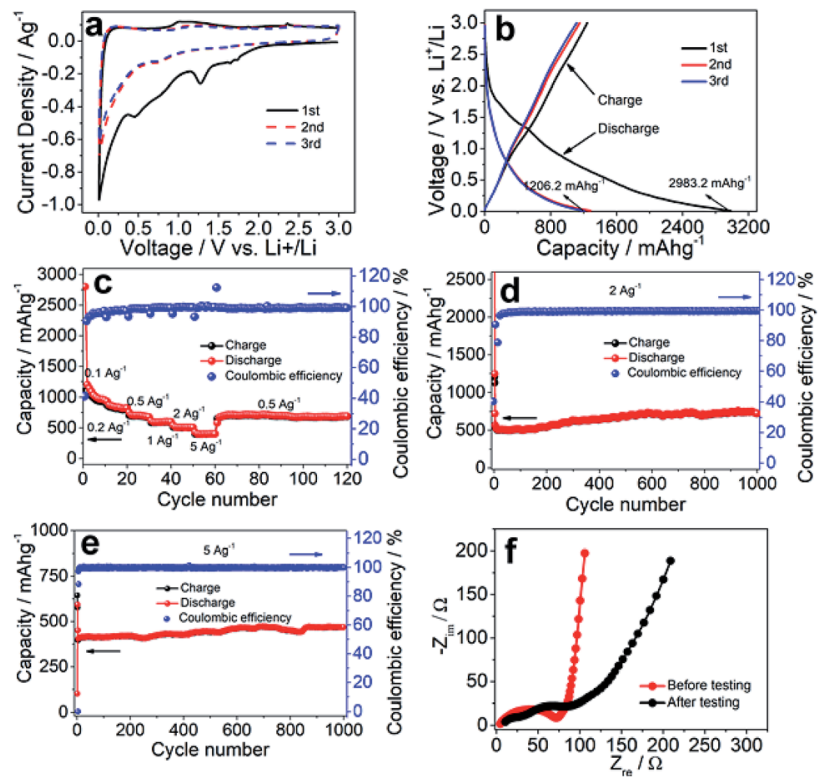

Fig. 7 The first three CV curves of NSHPC at $0.1 \mathrm{mV} \mathrm{s}^{-1}$ (a), the first three galvanostatic charge-discharge profiles at a current of $100 \mathrm{~mA} \mathrm{~g}^{-1}$ (b), and the rate performance (c), cycling performance at $2 \mathrm{~A} \mathrm{~g}^{-1}(\mathrm{~d})$, cycling performance at $5 \mathrm{~A} \mathrm{~g}^{-1}$ (e) of the assembled lithium ion battery using NSHPC as anode. (f) Nyquist plots of NSHPC before and after 1000 charge-discharge cycling at $5 \mathrm{~A} \mathrm{~g}^{-1}$. The potential window for cycling measurements is from 0.01 to $3.0 \mathrm{~V}\left(\mathrm{vs}\right.$. Li/ $\left.\mathrm{Li}^{+}\right)$. electrolyte decomposition, while the peak at about $1.3 \mathrm{~V}$ is possibly derived from Li-insertion into hydrogen atom-bonded carbon lattices. ${ }^{38}$ The other two weak peaks observed in the potential range of 1.5-2.2 $\mathrm{V}$ should be related to the Li trapped in the irreversible sites that is associated with high temperature induced collapse of the pores. ${ }^{39}$ The cathodic peaks in 0.01$0.3 \mathrm{~V}$ and in $0.7-1.0 \mathrm{~V}$ can be attributed to the Li-insertion into carbon matrix, and the lithium ion storage on the surface, respectively. The anodic peak appeared for all the three scans at about $1.1 \mathrm{~V}$ is associated with $\mathrm{Li}$ extraction from porous and defective sites of carbon matrix. ${ }^{40}$ The first three chargedischarge curves at the current density of $100 \mathrm{~mA} \mathrm{~g}^{-1}$ are shown in Fig. 7b. For the first discharge curve, several voltage plateaus are observed, corresponding to cathodic peaks on the CV curve of the first cathodic scan, which disappeared in the subsequent discharge process. An initial capacity of $2983 \mathrm{~mA} \mathrm{~h} \mathrm{~g}^{-1}$ was achieved from the first discharge curve and a reversible specific capacity of $1206 \mathrm{~mA} \mathrm{~h} \mathrm{~g}^{-1}$ was obtained with a coulombic efficiency of nearly $100 \%$, much higher than the theoretical value of battery using graphite anode $\left(372 \mathrm{~mA} \mathrm{~h} \mathrm{~g}{ }^{-1}\right){ }^{41}$ The observed large irreversible capacity can be attributed to the large specific surface induced SEI layer formation. ${ }^{42}$ Fig. $7 \mathrm{c}$ shows the rate performance of battery using NSHPC as anode, for which the cells were cycled at each rate for 10 cycles until a rate of $5 \mathrm{~A} \mathrm{~g}^{-1}$ was obtained, and then back to the current density of $0.5 \mathrm{~A} \mathrm{~g}^{-1}$ for 60 cycles. It is evident that the assembled battery exhibits an excellent rate performance. The cycling stability of the assembled battery using NSHPC as anode was investigated under both 2 and $5 \mathrm{~A} \mathrm{~g}^{-1}$ and the results were plotted in Fig. $7 \mathrm{~d}$ and e. It can be seen that the reversible discharge capacity of the assembled battery even slightly increased after 1000 cycles, from 420 to $470 \mathrm{~mA} \mathrm{~h} \mathrm{~g}^{-1}$ at $5 \mathrm{~A} \mathrm{~g}^{-1}$ and from 580 to $740 \mathrm{~mA} \mathrm{~h} \mathrm{~g}^{-1}$ at $2 \mathrm{~A} \mathrm{~g}^{-1}$, respectively. Such an increase in discharge capacity of the assembled battery could be explained by the further development of an SEI layer and the further activation of the porous anode. ${ }^{43}$ In addition, the charge-discharge behavior under relatively high current density is very similar to an ultrafast capacitor-like electrochemical response. ${ }^{44}$ The superior performance of NSHPC-based battery can be understood by considering the unique structural features and the synergistic effects of $\mathrm{N}$ and $\mathrm{S}$ co-doping. The interconnected porous structure can shorten the solid-state diffusion length of lithium ions in the bulk electrode while the large surface area of porous carbons can offer relatively large electrode/electrolyte interfaces that promote rapid charge transfer reactions in lithium ion batteries. ${ }^{40,45}$ Moreover, the high electronegativity of the codoped $\mathrm{N}$ and $\mathrm{S}$ atoms can synergistically lead to the structural distortion and the change of charge density of carbon framework, resulting in the attraction of larger number of lithium ions via electrostatic interactions compared to pristine carbon frameworks. ${ }^{46,47}$ To further understand the cyclability of the assembled battery, electrochemical impedance spectroscopy (EIS) measurements were recorded before and after the 1000 charge-discharge cycles at a current density of $5 \mathrm{~A} \mathrm{~g}^{-1}$ and the according Nyquist plots were displayed in Fig. 7f. In a type Nyquist plot, the semicircle in the high-medium frequency is attributed to summation of the contact resistance, the SEI 
resistance, and the charge-transfer resistance while the inclined line corresponds to the characteristic Warburg behavior related to the mass transfer resistance in the lithium-diffusion processing within carbon electrodes. ${ }^{44}$ The relatively small diameter of the semicircle diameter indicates that the synthesized NSHPC possesses a high electrical conductivity and a rapid charge transfer reaction for lithium ion insertion and extraction. The slight increase in the resistance after 1000 chargedischarge cycles under $5 \mathrm{~A} \mathrm{~g}^{-1}$ demonstrates that the thusassembled battery exhibits an excellent high-rate cyclability for $\mathrm{Li}$ insertion and extraction. ${ }^{\mathbf{4 1}}$

Since the synthesized NSHPC possesses a relatively large surface area with interconnected hierarchically porous structure (coexistence of micropores and mesopores), we finally investigated the electrochemical properties of NSHPC as electrode materials for supercapacitor in a three-electrode system using $6 \mathrm{~mol} \mathrm{~L}^{-1} \mathrm{KOH}$ aqueous solution as electrolyte. Fig. 8a shows the accordingly recorded CV curves of NSHPC. It can be seen that the CV curve shows nearly symmetrical rectangular shapes at the scan rate of $5 \mathrm{mV} \mathrm{s}^{-1}$. With increasing the scan rate to $200 \mathrm{mV} \mathrm{s}^{-1}$, NSHPC still keeps a rather good rectangularlike CV curve, which can be ascribed to the unique structural features of NSHPC. ${ }^{\mathbf{4 8 , 4 9}}$ Galvanostatic charge/discharge curves of NSHPC in Fig. 8b exhibit the symmetric shapes without obvious IR drop, demonstrating its small resistance, great reversibility, and high coulombic efficiency. ${ }^{50}$ At the current density of $1 \mathrm{~A} \mathrm{~g}^{-1}$, the specific capacitance of $203 \mathrm{~F} \mathrm{~g}^{-1}$ was achieved and the value is much higher than that of commercial ACs $(<100 \mathrm{~F}$ $\left.\mathrm{g}^{-1}\right) .{ }^{51}$ Even at the current densities of 7.5 and $10 \mathrm{~A} \mathrm{~g}^{-1}$, the NSHPC still retained the high capacitance values of 135.6 and 132.5 $\mathrm{F} \mathrm{g} \mathrm{g}^{-1}$, respectively (Fig. 8c). Moreover, the specific capacitance remained about $100 \%$ of its initial value after 5000 cycles at a current density of $5 \mathrm{~A} \mathrm{~g}^{-1}$ (Fig. 8d), revealing its extraordinarily high stability.
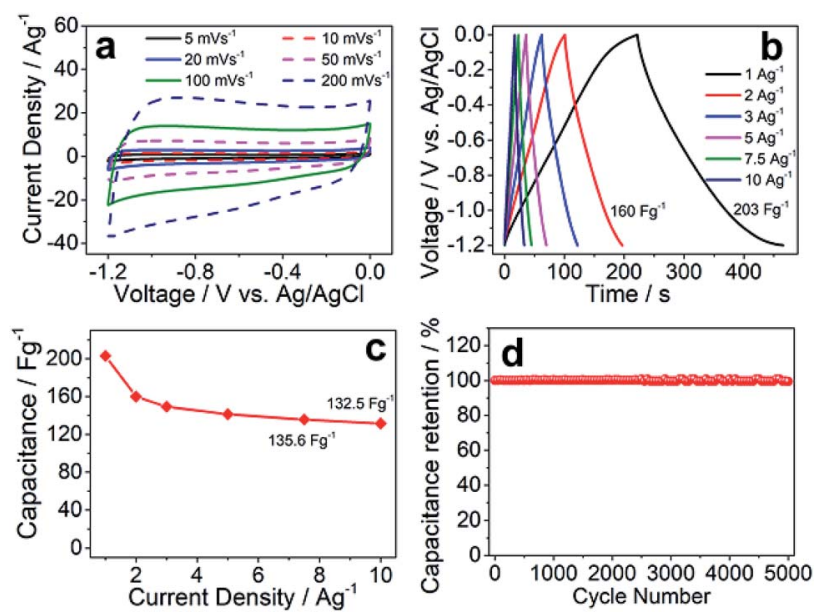

Fig. $8 \mathrm{CV}$ curves at the scan rates from 5 to $200 \mathrm{mV} \mathrm{s}^{-1}$ (a), galvanostatic charge-discharge curves at various current densities (b), and gravimetric capacitances at different charge-discharge current densities (c) of the assembled super capacitor using NSHPC as electrode in $6 \mathrm{~mol} \mathrm{~L}^{-1} \mathrm{KOH}$ aqueous solution. (d) Cycling performance at $2 \mathrm{~A} \mathrm{~g}^{-1}$ of the assembled supercapacitor using NSHPC as electrode.

\section{Conclusions}

We have demonstrated a new approach for synthesis of nitrogen, sulphur co-doped three-dimensional interconnected hierarchically porous carbon with a large specific surface area by zinc acetate assisted pyrolysis of polymer networks with welldistributed silica nanoparticles, followed by removal of silica templates. The evaporation of zinc acetate at high temperature can lead to the generation of micropores of the carbon materials while the crosslinked structure of polymer precursors improves the distribution of mesopores induced by silica template and increases the production yield of the resulted carbon materials. Benefiting from the unique structural features and the synergistic effect of nitrogen, sulphur co-doping, the resulted metalfree material has a large number of Lewis basic active sites for electrocatalysis as evidenced by carbon dioxide adsorption results and accordingly exhibits great electrocatalytic activity towards oxygen reduction reaction. Furthermore, the interconnected structure and the large surface area can shorten the solid-state diffusion length of lithium ions and can promote rapid charge transfer reactions, resulting in a good rate performance and cyclability of the thus-assembled lithium ion battery. Moreover, both the large specific surface area and the co-existence of micro- and meso-pores guarantee the high performance of the assembled supercapacitor including the specific capacitance and the stability. We believe that the simple and efficient synthetic strategy developed here may provide a new idea for design carbon-based electrocatalysts for multifunctional applications.

\section{Conflicts of interest}

There are no conflicts to declare.

\section{Acknowledgements}

This work was supported by the Natural Science Foundation of China under grant numbers of 21576216 and 21878239, International Cooperation and Exchanges Projects of Hubei Province (2017AHB057), and the Fundamental Research Funds for the Central Universities (2019-zy-016).

\section{Notes and references}

1 Y. Zhai, Y. Dou, D. Zhao, P. F. Fulvio, R. T. Mayes and S. Dai, Adv. Mater., 2011, 23, 4828.

2 B. Xia, Y. Yan, X. Wang and X. W. Lou, Mater. Horiz., 2014, 1, 379.

3 Z. Yang, H. Nie, X. Chen, X. Chen and S. Huang, J. Power Sources, 2013, 236, 238.

4 L. Wang, Z. Yang, H. Nie, C. Gu, W. Hua, X. Xu, X. Chen, Y. Chen and S. Huang, J. Mater. Chem. A, 2016, 4, 15343.

5 X. Zhou, J. Qiao, L. Yang and J. Zhang, Adv. Energy Mater., 2014, 4, 1301523.

6 L. Wei, M. Sevilla, A. B. Fuertes, R. Mokaya and G. Yushin, Adv. Funct. Mater., 2012, 22, 827. 
7 X. Zhou, Z. Tian, J. Li, H. Ruan, Y. Ma, Z. Yang and Y. Qu, Nanoscale, 2014, 6, 2603.

8 D. S. Su and R. Schlögl, ChemSusChem, 2010, 3, 136.

9 Q. Li, R. Cao, J. Cho and G. Wu, Adv. Energy Mater., 2014, 4, 1301415.

10 D. C. Higgins, M. A. Hoque, F. Hassan, J. Y. Choi, B. Kim and Z. Chen, ACS Catal., 2014, 4, 2734.

11 Q. Shi, F. Peng, S. Liao, H. Wang, H. Yu, Z. Liu, B. Zhang and D. Su, J. Mater. Chem. A, 2013, 1, 14853.

12 K. Gong, F. Du, Z. Xia, M. Durstock and L. Dai, Science, 2009, 323, 760.

13 J. Zhang, Z. Zhao, Z. Xia and L. Dai, Nat. Nanotechnol., 2015, 10, 444 .

14 Z. Yang, Z. Yao, G. Li, G. Fang, H. Nie, Z. Liu, X. Zhou, X. Chen and S. Huang, ACS Nano, 2012, 6, 205.

15 D. W. Wang, F. Li, M. Liu, G. Q. Lu and H. M. Cheng, Angew. Chem., Int. Ed., 2008, 47, 373.

16 Z. Yang, J. Ren, Z. Zhang, X. Chen, G. Guan, L. Qiu, Y. Zhang and H. Peng, Chem. Rev., 2015, 115, 5159.

17 C. Zhao, C. Yu, S. Liu, J. Yang, X. Fan, H. Huang and J. Qiu, Adv. Funct. Mater., 2016, 25, 6913.

18 Z. Wu, W. Ren, L. Xu, F. Li and H. Cheng, ACS Nano, 2011, 5, 5463.

19 Z. Liu, H. Nie, Z. Yang, J. Zhang, Z. Jin, Y. Lu, Z. Xiao and S. Huang, Nanoscale, 2013, 5, 3283.

20 J. Liang, Y. Jiao, M. Jaroniec and S. Z. Qiao, Angew. Chem., Int. Ed., 2012, 51, 11496.

21 P. A. Denis, R. Faccio and A. W. Mombru, ChemPhysChem, 2009, 10, 715.

22 P. A. Denis, C. P. Huelmo and F. Iribarne, Comput. Theor. Chem., 2014, 1049, 13.

23 J. Wang, X. Li, M. Fan, H. Zhang and W. Tu, ChemistrySelect, 2018, 3, 3006.

24 G. S. Chai, I. S. Shin and J. S. Yu, Adv. Mater., 2004, 16, 2057.

25 A. Li, Y. Tong, B. Cao, H. Song, Z. Li, X. Chen, J. Zhou, G. Chen and H. Luo, Sci. Rep., 2017, 7, 40574.

26 Y. Pan, K. Sun, S. Liu, X. Cao, K. Wu, W. Cheong, Z. Chen, Y. Wang, Y. Li, Y. Liu, D. Wang, Q. Peng, C. Chen and Y. Li, J. Am. Chem. Soc., 2018, 140, 2610.

27 Z. Xu, X. Zhuang, C. Yang, J. Cao, Z. Yao, Y. Tang, J. Jiang, D. Wu and X. Feng, Adv. Mater., 2016, 28, 1981.

28 J. Zhang, Y. Sun, J. Zhu, Z. Kou, P. Hu, L. Liu, S. Li, S. Mu and Y. Huang, Nano Energy, 2018, 52, 307.

29 J. Zhi, W. Zhao, X. Liu, A. Chen, Z. Liu and F. Huang, Adv. Funct. Mater., 2014, 24, 2013.
30 G. Zhou, E. Paek, G. S. Hwang and A. Manthiram, Nat. Commun., 2015, 6, 7760.

31 T. Sun, J. Wang, C. Qiu, X. Ling, B. Tian, W. Chen and C. Su, Adv. Sci., 2018, 5, 1800036.

32 H. Wang, C. Zhang, Z. Liu, L. Wang, P. Han, H. Xu, K. Zhang, S. Dong, J. Yao and G. Cui, J. Mater. Chem., 2011, 21, 5430.

33 D. Li, Y. Jia, G. Chang, J. Chen, H. Liu, J. Wang, Y. Hu, Y. Xia, D. Yang and X. Yao, Chem, 2018, 4, 2345.

34 I. S. Amiinu, J. Zhang, Z. Kou, X. Liu, O. K. Asare, H. Zhou, K. Cheng, H. Zhang, L. Mai, M. Pan and S. Mu, ACS Appl. Mater. Interfaces, 2016, 8, 29408.

35 D. Guo, R. Shibuya, C. Akiba, S. Saji, T. Kondo and J. Nakamura, Science, 2016, 351, 361.

36 A. Aijaz, N. Fujiwara and Q. Xu, J. Am. Chem. Soc., 2014, 136, 6790.

37 X. Li, F. Zhang, J. Yuan, H. Zhang, M. Pan, C. Huang and H. Tang, ChemNanoMat, 2016, 2, 1028.

38 L. G. Bulusheva, A. V. Okotrub, A. G. Kurenya, H. Zhang, H. Zhang, X. Chen and H. Song, Carbon, 2011, 49, 4013.

39 W. Ai, J. Jiang, J. Zhu, Z. Fan, Y. Wang, H. Zhang, W. Huang and T. Yu, Adv. Energy Mater., 2015, 5, 1500559.

40 Z. Tan, K. Ni, G. Chen, W. Zeng, Z. Tao, M. Ikram, Q. Zhang, H. Wang, L. Sun, X. Zhu, X. Wu, H. Ji, R. S. Ruoff and Y. Zhu, Adv. Mater., 2017, 29, 1603414.

41 S. Yang, X. Feng, L. Zhi, Q. Cao, J. Maier and K. Müllen, Adv. Mater., 2010, 22, 838.

42 Z. Wang, F. Li, N. S. Ergang and A. Stein, Chem. Mater., 2006, 18, 5543.

43 Y. Wang, H. Wei, Y. Lu, S. Wei, E. K. Wujcik and Z. Guo, Nanomaterials, 2015, 5, 755.

44 X. Yu, C. Zhan, R. Lv, Y. Bai, Y. Lin, Z. H. Huang, W. Shen, X. Qiu and F. Kang, Nano Energy, 2015, 15, 43.

45 M. S. Kim, D. Bhattacharjya, B. Fang, D. S. Yang, T. S. Bae and J. S. Yu, Langmuir, 2013, 29, 6754.

46 W. Ai, Z. Luo, J. Jiang, J. Zhu, Z. Du, Z. Fan, L. Xie, H. Zhang, W. Huang and T. Yu, Adv. Mater., 2014, 26, 6186.

47 W. H. Shin, H. M. Jeong, B. G. Kim, J. K. Kang and J. W. Choi, Nano Lett., 2012, 12, 2283.

48 F. Béguin, V. Presser, A. Balducci and E. Frackowiak, Adv. Mater., 2014, 26, 2219.

49 Y. Li, Z. Li and P. K. Shen, Adv. Mater., 2013, 25, 2474.

50 J. Zhao, H. Lai, Z. Lyu, Y. Jiang, K. Xie, X. Wang, Q. Wu, L. Yang, Z. Jin, Y. Ma, J. Liu and Z. Hu, Adv. Mater., 2015, 27, 3541.

51 C. Long, L. Jiang, X. Wu, Y. Jiang, D. Yang, C. Wang, T. Wei and Z. Fan, Carbon, 2015, 93, 412. 